\title{
P-Doped Porous Carbon as Metal Free Catalysts for Selective Aerobic Oxidation with an Unexpected Mechanism
}

Mehulkumar A. Patel,$\uparrow$ Feixiang Luo, $†$ M. Reza Khoshi, $\dagger$ Emann Rabie, $\dagger$ Qing Zhang, $\S$ Carol R. Flach, $\dagger$ Richard Mendelsohn, $\uparrow$ Eric Garfunkel, + Michal Szostak, $* \dagger$ and Huixin He, $* \dagger$

$\dagger$ Department of Chemistry, Rutgers University, 73 Warren Street, Newark, New Jersey 07102, United States

†Department of Chemistry and Chemical Biology, Rutgers University, 610 Taylor Rd, Piscataway, NJ 08854, USA

§Department of Chemistry, Jilin University, 2699 Qianjin Street, Changchun 130012, P. R. China.

\section{Supporting Information}

\section{Experimental section:}

\section{PGc (Phosphorus doped graphitic carbon) fabrication:}

The phytic acid (Sigma Aldrich, $50 \mathrm{w} / \mathrm{w} \%$ in water, $1 \mathrm{~mL}$ ) was placed in $35 \mathrm{~mL}$ Pyrex glass vessel (CEM, \#909036) and then closed with Teflon lined cap (CEM, \#909235). This closed glass vessel was kept in $500 \mathrm{~mL}$ beaker and then the whole assembly was covered with watch glass before transferring to a domestic microwave oven $(1100 \mathrm{~W}$, Sanyo-EM-S9515W, $2.45 \mathrm{GHz}$ ). A microwave irradiation was applied for 40 seconds which results into black carbonized material. After microwave treatment, the glass tube was left in a fume hood for a few minutes to remove any gas generated during microwave reaction and then dispersed in ethanol by bath sonication ( 5 minutes). The resulting dispersion is filtered by $0.8 \mu \mathrm{m}$ polycarbonate filter paper (Millipore, ATTP 04700) and washed with water $(\sim 800 \mathrm{~mL})$ and ethanol $(\sim 400 \mathrm{~mL})$. After filtration, the product was dried in vacuum oven at $\sim 110^{\circ} \mathrm{C}$ overnight before further use. The yield of product (PGc) was calculated to be $\sim 12 \%$ by the weight of pure phytic acid or $\sim 90 \%$ by weight of carbon present in phytic acid. Note: The above fabrication reaction can be also carried out in single mode cavity using commercial CEM microwave (CEM Discover SP, 300 Watts). This microwave unit provides much higher energy density than domestic microwave and so PGc can be synthesized in shorter time (30 seconds at 300 watts) than domestic microwave. It is also worthy to mention that the bulk price of phytic acid is $\$ 0.03$ per gram, which is much cheaper compared to GO ( $\$ 200$ per gram) and it is also sustainable for synthesis of $\mathrm{P}$ doped carbon materials. 


\section{Fabrication of PGc-30 and PGc-180:}

The PGc powder was further heated in the same microwave oven with additional microwave irradiation of total $30 \mathrm{~s}$ and $180 \mathrm{~s}$, respectively. In detail, $\sim 100 \mathrm{mg}$ of PGc powder weighed into small porcelain dish and covered with a piece of watch glass before transferring into the domestic microwave chamber. The microwave radiation was applied in pulse of 10 seconds for different times with a 10-15 minute interval between two microwave pulses to avoid over heating or burning of the carbon material. For example, to synthesize PGc-180, 10 seconds of microwave radiation was repeated for 18 times with 10-15 minutes interval in between each microwave pulse.

\section{Synthesis of GO and rGO for catalysis:}

GO is synthesized according to Hummer's method with slight modification. ${ }^{1}$ In brief, the graphite powder (2 g, Sigma Aldrich, $<20 \mu \mathrm{m}$ ) is mixed with $55 \mathrm{~mL}$ sulfuric acid (PHARMCO-AAPER, ACS grade 95-98\%) and stirred in ice bath for $15 \mathrm{~min}$. After that we added $12 \mathrm{~mL}$ nitric acid (BDH, ACS grade 69-70\%) and again stirred in ice bath for additional 15 minute to cool down the mixture. Then we added $10 \mathrm{~g}$ of KMnO4 $(10 \mathrm{~g}$, Sigma Aldrich, ACS grade) in a small portions while stirring the mixture in ice bath. After 2 hours of stirring in ice bath, the mixture was stirred in water bath at $45{ }^{\circ} \mathrm{C}$ for 6 hours to complete the oxidation of graphite. After Reaction was completed, it was quenched in $500 \mathrm{~mL}$ ice containing $10 \mathrm{~mL}$ of $\mathrm{H}_{2} \mathrm{O}_{2}(\mathrm{BDH}, 35 \mathrm{w} / \mathrm{w} \%)$ and filtered using what man filter paper (grade 5, $47 \mathrm{~mm}$ ). Then the brown solid powder was resuspended in $\sim 4 \% \mathrm{HCl}$ and washed with it for 5 times by centrifugation at $8000 \mathrm{rpm} * 30$ minutes. After that it was washed with acetone for 10 times at $10000 \mathrm{rpm}^{*} 45$ minutes of centrifugation and then dried in vacuum oven for 3 days before further use.

To synthesize rGO, $500 \mathrm{mg}$ of GO was taken in the round bottom flask and then heated with microwave of 300 Watt (CEM discover SP) for 40secs. The brown colored GO powder was converted to black colored reduced graphene oxide (rGO) which was used as a control in catalytic reaction of benzyl alcohol oxidation to benzaldehyde in water solvent.

\section{Catalytic oxidation of primary and secondary alcohol Reaction:}

All the chemicals were used as received for catalytic reaction. Benzyl Alcohol (Millipore, $\geq 99 \%$ ) and DL-sec-phenyl ethyl alcohol (Acros Organics, $\geq 97 \%$ ), Cyclohexane methanol (Alfa Aesar, 99\%), n butanol (anhydrous, Sigma Aldrich, 99.8\%), 4-methoxybenzyl alcohol (TCI, >98\%), 4-methylbenzyl alcohol (Sigma Aldrich, 98\%), 4-nitrobenzyl alcohol (Alfa Aesar, 99\%), Toluene (anhydrous, Sigma Aldrich, 99.8\%) Ethyl benzene (Alfa Aesar, 99\%), tert-butyl hydroperoxide (TBHP) (Alfa Aesar, 70\%), $\mathrm{H}_{2} \mathrm{O}_{2}(\mathrm{BDH}, 35 \% \mathrm{w} / \mathrm{w})$. 
Alcohol oxidation in water: The aerobic oxidation reactions were carried out in a round bottom flask or $35 \mathrm{ml}$ reaction tube (depending on the size of the reaction) by stirring water, alcohol and catalyst under $1 \mathrm{~atm}$ oxygen environment (using oxygen balloon). The detailed experimental condition and the amount of reagent and catalyst are specified in Table-1 footnote. After the reaction is completed, reaction mixture is filtered via $0.02 \mu \mathrm{m}$ syringe filter and analyzed by HPLC (Varian Pro-Star and Phenomenex C18 column, mobile phase 50:50 ratio of Methanol: $0.44 \%$ Acetic acid). For kinetic studies, the experiment was carried out at different temperatures. During each of the experiments, $\sim 0.3 \mathrm{~mL}$ of aliquot was withdrawn at a regular interval of 15 minutes, filtered via 0.02 $\mu \mathrm{m}$ syringe filter and analyzed by HPLC.

Solvent free alcohol oxidation: In a typical reaction, benzyl alcohol was purged with oxygen for 10 minutes prior to mixing with a specified amount of catalyst (as mentioned in Table 2) in a microwave reaction vial (VWR, 10-20 mL, \#89079-402) and sealed with PTFE faced aluminum cap. The reaction vial was heated in oil bath at specified temperature and time. For controlled experiment, nitrogen gas was used instead of oxygen. For control experiment with a radical inhibitor, BHT (Butylated hydroxytoluene), specified amount of BHT and acetonitrile (for maintaining uniform dispersion of BHT) was added to the above described mixture in the beginning of the reaction. For alcohol oxidation using TBHP or $\mathrm{H}_{2} \mathrm{O}_{2}$ as oxidant, TBHP or $\mathrm{H}_{2} \mathrm{O}_{2}$ was mixed with the specific alcohol and catalysts and then sealed in ambient environment. The experimental condition and the amount of the reactant and catalysts are specified in the table footnote. For control experiment using P-OH functional containing molecules (such as phytic acid or phosphoric acid) as a catalyst, we have mixed $1 \mathrm{mmol}$ of benzyl alcohol and catalyst such that mmols of $\mathrm{P}-\mathrm{OH}$ functional group comes to $1.2 \mathrm{mmols}$ and heated under $80{ }^{\circ} \mathrm{C}$ for 48 hours under 1 atm $\mathrm{O}_{2}$. After completion of the reaction, $\sim 0.7$ $\mathrm{mL}$ of $\mathrm{CDCl}_{3}$ was mixed with the reaction mixture and filtered via $0.02 \mu \mathrm{m}$ syringe filter and analyzed by ${ }^{1} \mathrm{H}$ NMR spectroscopy (Bruker Avalanche $500 \mathrm{MHz}$ ).

\section{Material Characterization:}

The PeakForce Kelvin probe force microscopy (PF-KPFM) measurements of the PGc and PGc-180 catalysts were conducted using a Dimension ICON AFM setup inside a nitrogen-filled glove box where both $\mathrm{H}_{2} \mathrm{O}$ and $\mathrm{O}_{2}$ level were below $0.1 \mathrm{ppm}$. The tips used were PFQNE-AL (Bruker AFM Probes), composed of a silicon nitride cantilever with a sharp silicon tip. The morphology of PGc samples were studied using the scanning electron microscopy (SEM, Hitachi S-4800). The sample for SEM was prepared by adding the powder sample on the carbon tape. X-ray photoelectron spectroscopy (XPS) characterization was performed after depositing a layer of the catalyst to be studied onto a Si substrate. The thickness of the film on the substrates was roughly $30-50 \mathrm{~nm}$. XPS spectra were acquired using a Thermo Scientific K-Alpha system with a monochromatic Al $\mathrm{K} \alpha$ x-ray source $(\mathrm{h} v=1486.7 \mathrm{eV})$. For data analysis, Smart background subtraction was performed, and the spectra were fit with Gaussian/Lorentzian peaks using a minimum deviation curve fitting method (part of the Avantage software package). The 
surface composition of each species was determined by the integrated peak areas and the Scofield sensitivity factor provided by the Avantage software. The Fourier transform infrared spectroscopy (FT-IR) spectra of the samples (thin films deposited on $\mathrm{ZnSe}$ windows) were acquired with a Thermo-Nicolet 6700 spectrometer (Thermo-Electron Corp., Madison, WI), using a sample shuttle and a mercury-cadmium-telluride (MCT) detector. Four blocks of 128 scans each were co-added with $4 \mathrm{~cm}^{-1}$ spectral resolution and two levels of zero-filling so that data was encoded every $1 \mathrm{~cm}^{-1}$. Raman spectra of the samples (deposited on Anodisc membrane) were collected using Raman Microscope (Confocal) - Wi-Tec, Alpha 3000R with an excitation laser at $785 \mathrm{~nm}$. Thermogravimetric analyses (TGA) of the PGc samples were performed on a TGA instrument (TA instruments, Discovery TGA) under $\mathrm{N}_{2}$ atmosphere. $\sim 5 \mathrm{mg}$ sample was loaded on to TGA platinum HT pan and kept at $40{ }^{\circ} \mathrm{C}$ for 5 minutes before each analysis. After 5 minutes, the temperature is increased from $40^{\circ} \mathrm{C}$ to $900^{\circ} \mathrm{C}$ at $5^{\circ} \mathrm{C} / \mathrm{min}$ under $\mathrm{N}_{2}$ flow $(20 \mathrm{ml} / \mathrm{min})$.

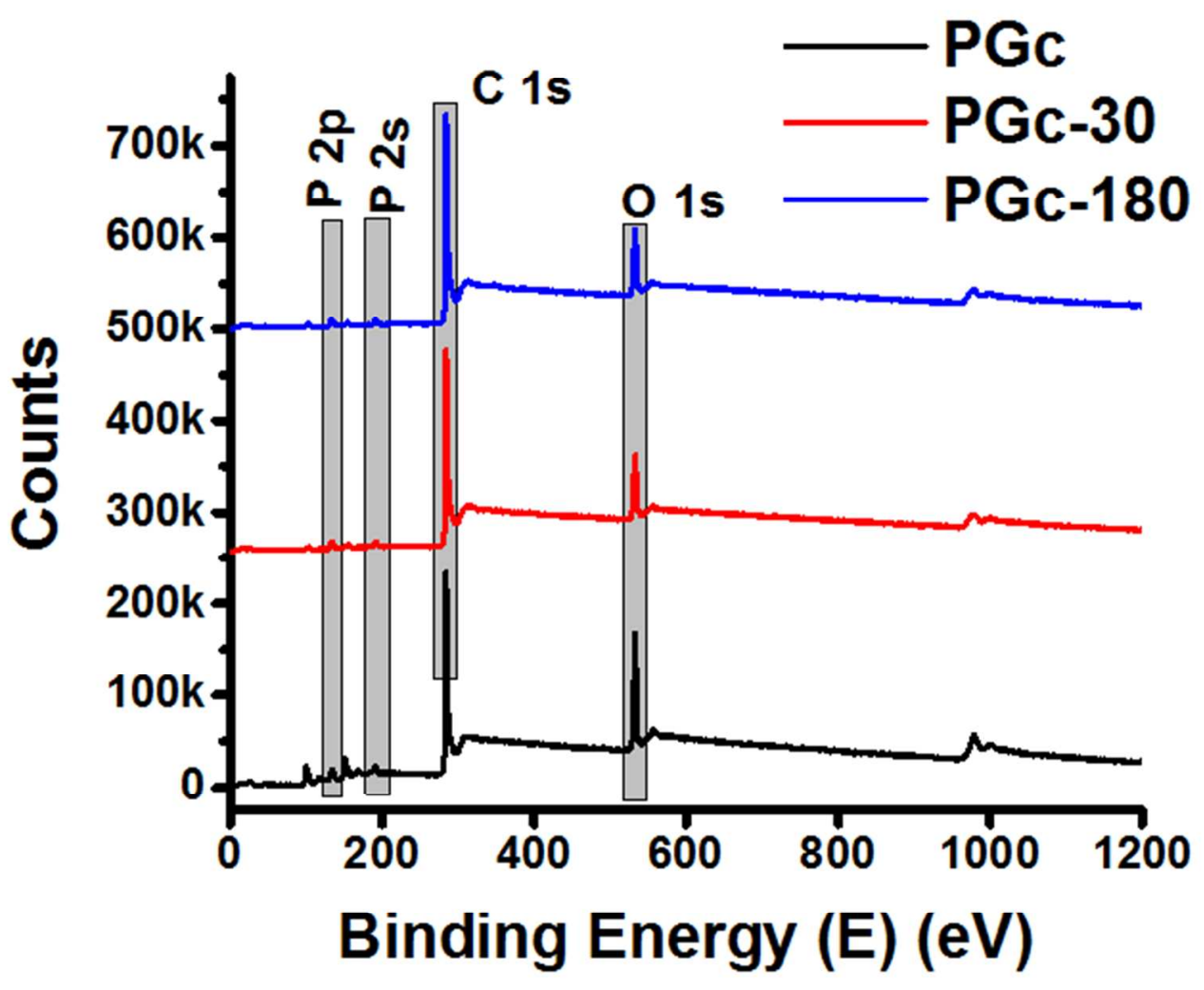

Figure S1. Survey XPS spectrum of PGc, PGc-30 and PGc-180 catalysts. 

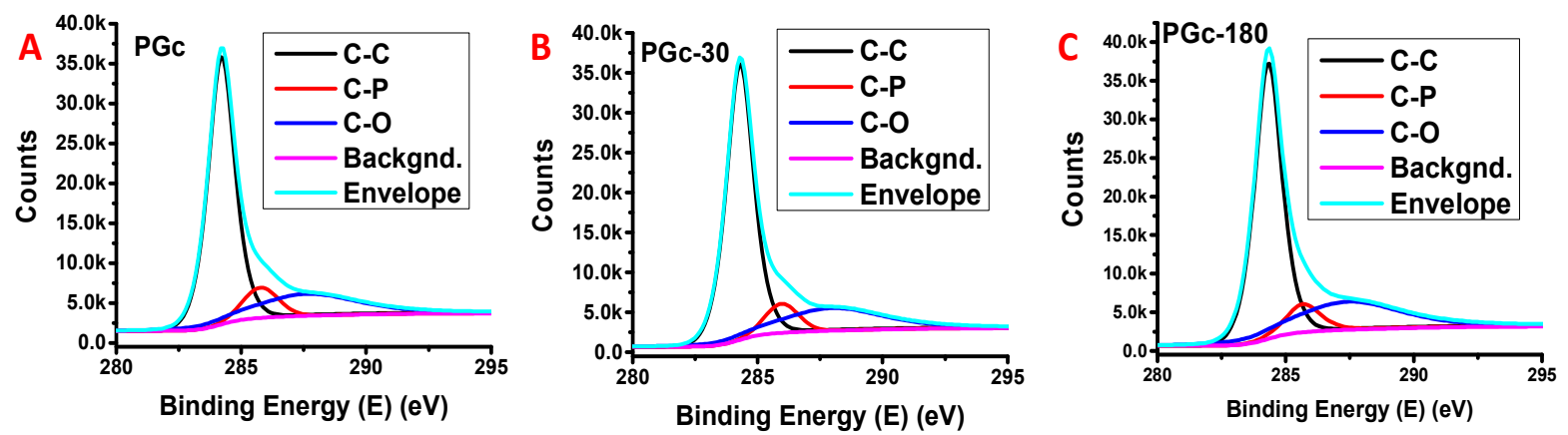

Figure S2. C 1s Peak deconvolution of different PGc catalysts, PGc (A), PGc-30 (B) and PGc$180(\mathrm{C})$.

Table S1.Calculated atomic \% of $\mathrm{C}, \mathrm{P}$ and $\mathrm{O}$ for PGc, PGc-30 and PGc-180 catalysts.

\begin{tabular}{|c|c|c|c|}
\hline Catalyst & \% C & \% O & \% P \\
\hline PGc & 73.94 & 21.17 & 4.89 \\
\hline PGc-30 & 83.26 & 13.52 & 3.21 \\
\hline PGc-180 & 83.56 & 13.60 & 2.84 \\
\hline
\end{tabular}

Table S2. Calculated \% of P-C and P-O present in PGc, PGc-30 and PGc-180 catalysts.

\begin{tabular}{|c|c|c|c|c|c|c|}
\hline & \multicolumn{3}{|c|}{ Absolute \% } & \multicolumn{3}{c|}{ Relative \% } \\
\hline Catalyst & $\%$ P-C & $\%$ P-O & Total & $\%$ P-C & $\%$ P-O & Total \\
\hline PGc-0 & 1.39 & 3.50 & 4.89 & 28.43 & 71.57 & 100 \\
\hline PGc-30 & 1.27 & 1.94 & 3.21 & 39.56 & 60.44 & 100 \\
\hline PGc-180 & 1.15 & 1.69 & 2.84 & 40.49 & 59.51 & 100 \\
\hline
\end{tabular}

Absolute $\%$ is calculated based on all the elements $(\mathrm{C}, \mathrm{P}$ and $\mathrm{O})$ in the material. Relative $\%$ is calculated based on total amount of $\mathrm{P}$ present. 
Table S3. Calculated \% of different type of oxygen present in PGc, PGc-30 and PGc-180 catalysts.

\begin{tabular}{|l|c|c|c|c|c|c|c|c|c|c|}
\hline & \multicolumn{5}{|c|}{ Absolute \% } & \multicolumn{5}{c|}{ Relative \% } \\
\hline Catalysts & $\begin{array}{c}\mathrm{C}=\mathrm{O} / \mathrm{P}= \\
\mathrm{O}\end{array}$ & $\begin{array}{c}\mathrm{C}-\mathrm{O} / \mathrm{P}- \\
\mathrm{O}-\mathrm{C}\end{array}$ & $\begin{array}{c}\mathrm{C}- \\
\mathrm{OH} / \mathrm{P}- \\
\mathrm{OH}\end{array}$ & $\begin{array}{c}\mathrm{COOH} / \\
\text { Water }\end{array}$ & Total & $\begin{array}{c}\mathrm{C}=\mathrm{O} / \mathrm{P}= \\
\mathrm{O}\end{array}$ & $\begin{array}{c}\mathrm{C}-\mathrm{O} / \mathrm{P}- \\
\mathrm{O}-\mathrm{C}\end{array}$ & $\begin{array}{c}\mathrm{C}- \\
\mathrm{OH} / \mathrm{P}- \\
\mathrm{OH}\end{array}$ & $\begin{array}{c}\mathrm{COOH} / \\
\text { Water }\end{array}$ & Total \\
\hline PGr-0sec & 2.22 & 3.38 & 13.25 & 2.32 & 21.17 & 10.49 & 15.97 & 62.57 & 10.96 & 99.99 \\
\hline PGr-30 & 2.17 & 3.42 & 6.78 & 1.15 & 13.52 & 16.05 & 25.30 & 50.15 & 8.51 & 100.01 \\
\hline PGr-180 & 2.14 & 4.45 & 5.88 & 1.13 & 13.60 & 15.74 & 32.72 & 43.24 & 8.31 & 100.01 \\
\hline
\end{tabular}

Absolute \% is calculated based on presence of all the elements (C, P and $\mathrm{O})$ in the material. Relative \% is calculated based on total amount of P present.

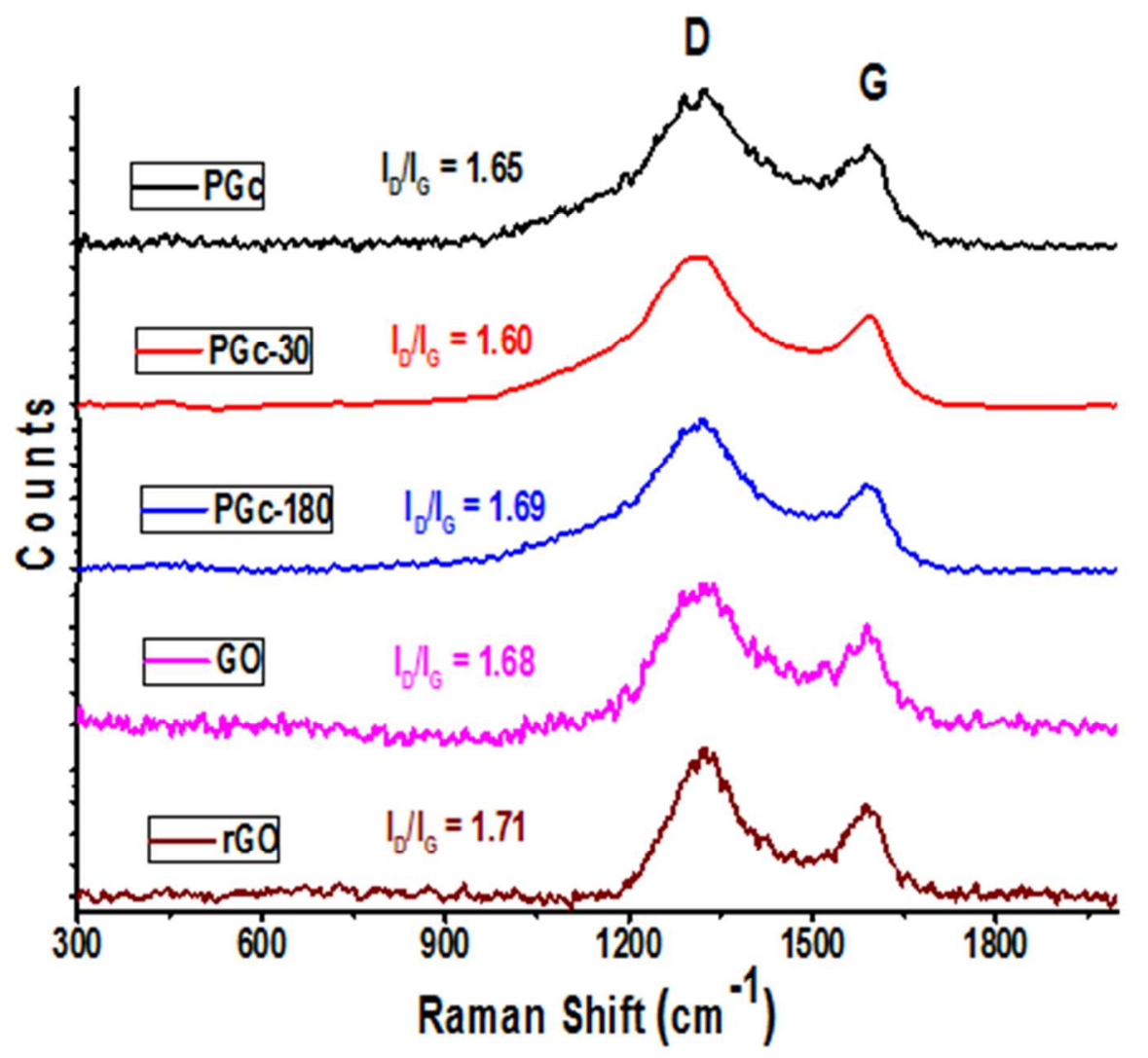

Figure S3. The Raman spectra of different catalysts. 


\section{The Surface area measurement by Brunauer-Emmett-Teller (BET) method:}

The surface areas of the PGc catalyst was determined using a 12-point BET method (Micromeritics, ASAP 2020) and nitrogen as the adsorbate using. ${ }^{2}$ After the BET measurements, the isotherms of these measurement are converted into BET plots as shown in Figure S1 and then the specific surface area of the catalyst was calculated using the value of the slope and intercept of the linear best fit line and below BET equation. ${ }^{2}$

$$
\frac{1}{Q\left[\left(P_{0} / P\right)-1\right]}=\frac{c-1}{Q_{\mathrm{m}} \mathrm{c}}\left(\frac{P}{P_{0}}\right)+\frac{1}{W_{\mathrm{m}} C}
$$

Here, $Q$ is adsorbed gas quantity, $Q_{\mathrm{m}}$ is monolayer quantity of adsorbed gas, c is the BET constant,

$P$ and $P_{0}$ are the equilibrium and the saturation pressure of adsorbates at the temperature of adsorption, respectively.

The Calculated surface area of the PGc catalyst is $1260 \mathrm{~m}^{2} / \mathrm{g}$.

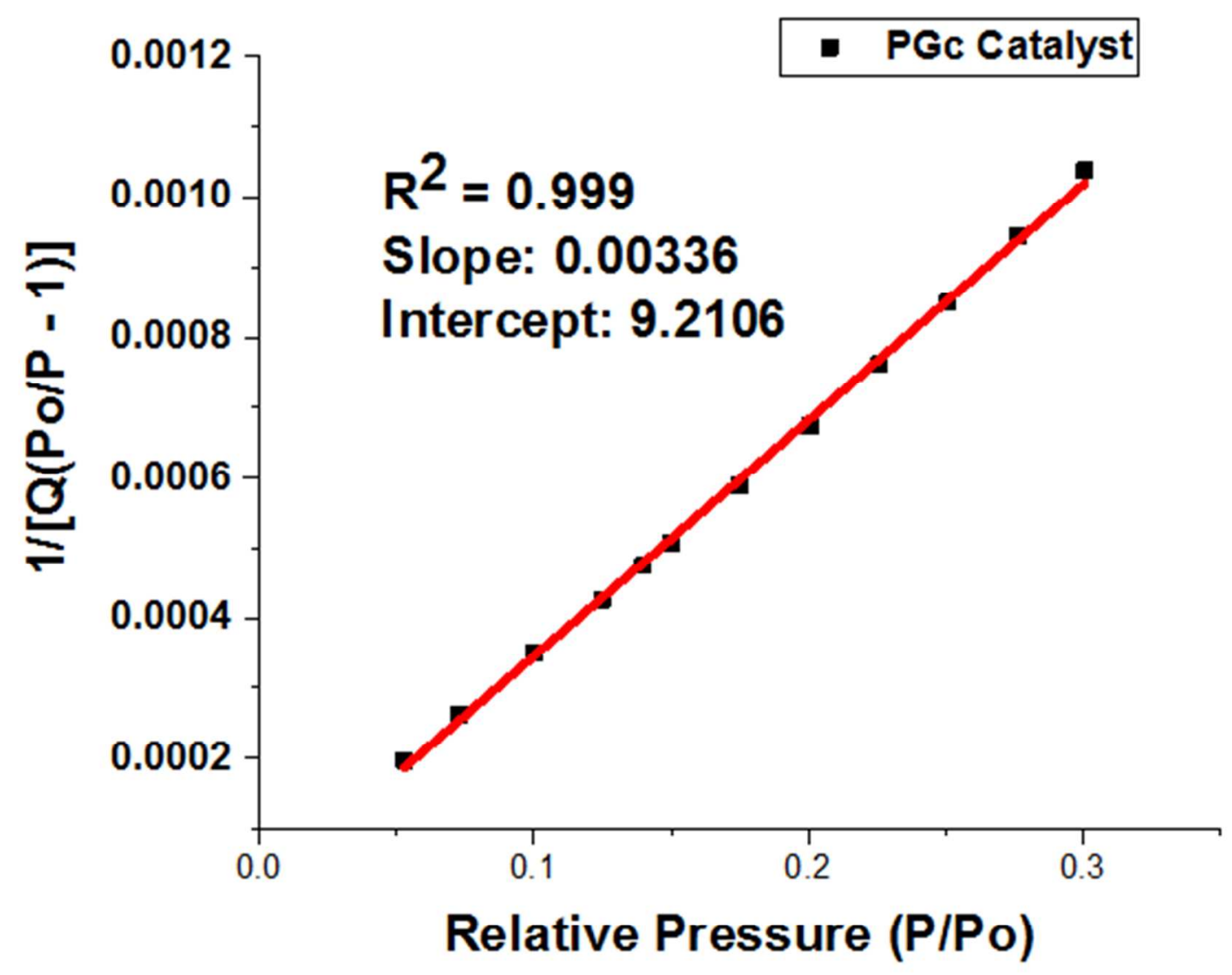

Figure S4. 12-point BET plot of PGc catalyst. 


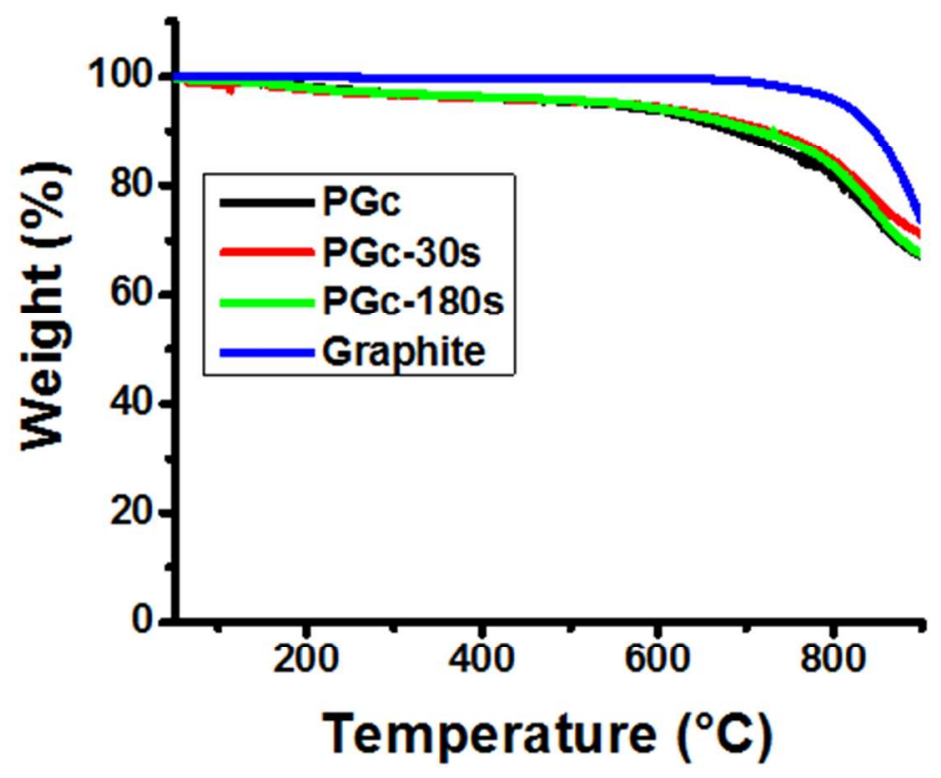

Figure S5. TGA (Thermo Gravimetric Analysis) spectra of different phosphorus doped carbon catalyst and graphite.
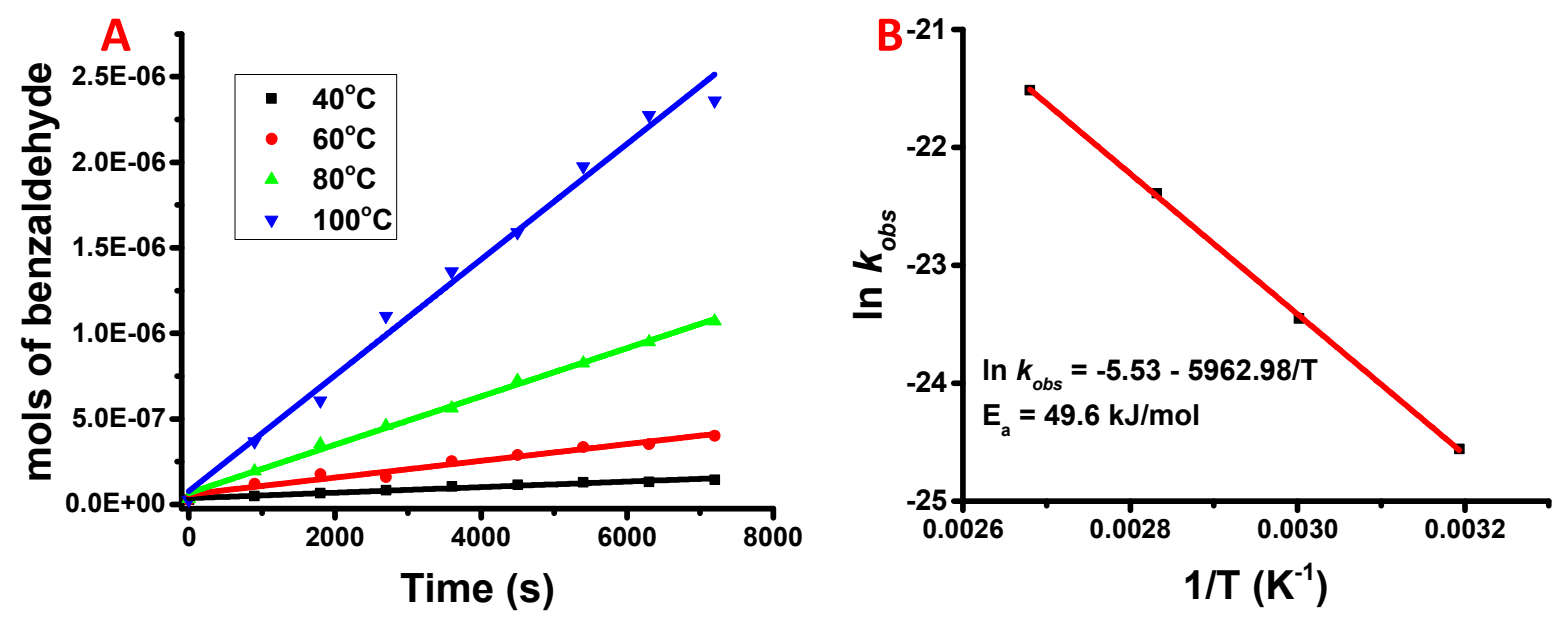

Figure S6. (A) Effects of reaction temperatures on the rate of oxidation of benzyl alcohol. Reaction conditions: $7 \mathrm{mg}$ benzyl alcohol, $10.5 \mathrm{mg}$ PGc catalyst, $10 \mathrm{ml}$ water, $1 \mathrm{~atm} \mathrm{O}_{2}$. (B) Arrhenius plot for the Benzyl alcohol oxidation. The rate constant $(\mathrm{k})$ values at different temperature were regarded as the pseudo-zero-order rate constants ( $\mathrm{k}$ obs $)$ because the plot of the mols of benzaldehyde produced versus time is linear. 
Table S4. The benzyl alcohol oxidation catalyzed by PGc in the presence of $\mathrm{H}_{2} \mathrm{O}_{2}$ and TBHP oxidants ${ }^{\text {a }}$

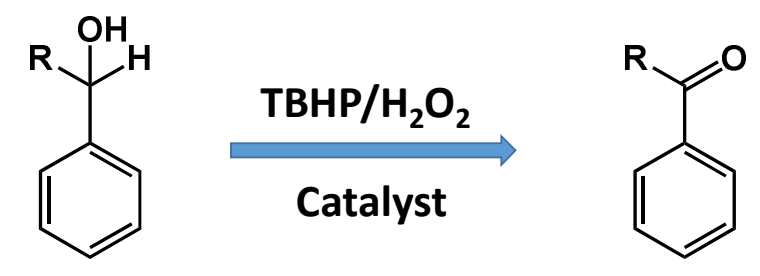

\begin{tabular}{|c|c|c|c|c|c|}
\hline Entry & $\begin{array}{c}\text { Catalyst, } \\
\text { Oxidant }\end{array}$ & $\mathbf{R}$ & $\begin{array}{c}\text { (\%) } \\
\text { Conversion }\end{array}$ & (\%) Selectivity & TON $\left(\mathrm{x} \mathrm{10}^{-2}\right)$ \\
\hline $\mathbf{1}$ & $\begin{array}{c}\text { No catalyst, } \\
\text { TBHP }\end{array}$ & $\mathrm{H}$ & 13.92 & 96.0 & - \\
\hline $\mathbf{2}$ & PGc, TBHP & $\mathrm{H}$ & 98.78 & $\begin{array}{c}0.01 \% \text { - Benzaldehyde } \\
99.99 \% \text { Benzoic acid }\end{array}$ & 5.02 \\
\hline $\mathbf{3}$ & PGc, TBHP & $\mathrm{CH}_{3}$ & 95.73 & $>99$ & 4.36 \\
\hline $\mathbf{4}$ & PGc, $\mathrm{H}_{2} \mathrm{O}_{2}$ & $\mathrm{H}$ & 27.74 & 68.7 & 1.41 \\
\hline $\mathbf{5}$ & PGc, $\mathrm{H}_{2} \mathrm{O}_{2}$ & $\mathrm{CH}_{3}$ & 27.25 & 92.1 & 1.24 \\
\hline
\end{tabular}

Reaction conditions- $10 \mathrm{mg}$ catalyst, $50 \mathrm{mg}$ alcohol $(0.5 \mathrm{mmol})$, oxidant-TBHP/ $\mathrm{H}_{2} \mathrm{O}_{2}(1.5$ $\mathrm{mmol}), 80^{\circ} \mathrm{C}, 24$ hours. Acetonitrile added as a solvent to make final reaction volume $0.3 \mathrm{ml}$ in all reactions. \% conversion for alcohols and \% selectivity to benzaldehyde calculated using $1 \mathrm{H}$ NMR. The turnover number (TON) was calculated as a ratio of the (mol of oxidized product) / (mass of catalyst). 


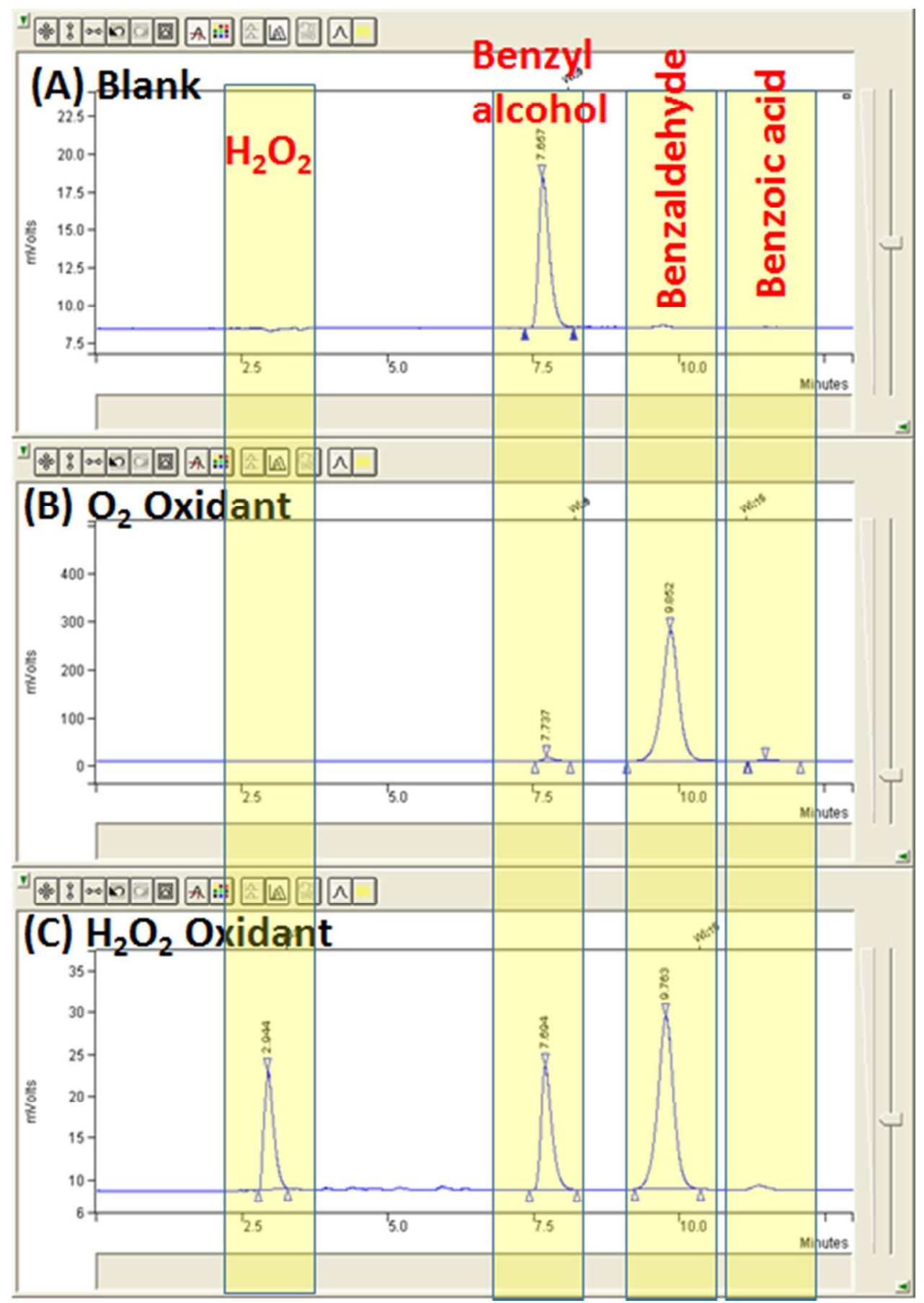

Figure S7. HPLC chromatogram of blank (No catalyst), PGe catalyst with an oxygen oxidant (B) and PGc catalyst with an $\mathrm{H}_{2} \mathrm{O}_{2}$ oxidant(C). Reaction condition for (A) and (B) can be found in Table 2- entry no. 1 and 4. Reaction condition for (C) can be found in Table S4 entry no. 4. 


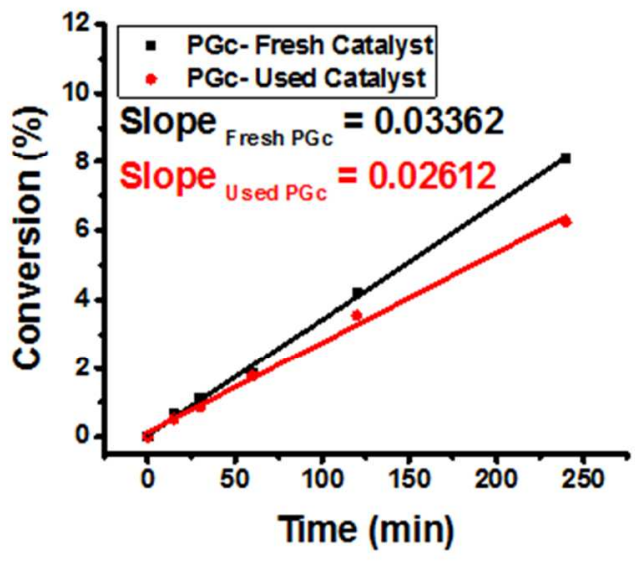

Figure S8. Time conversion plot of a fresh and used PGc catalyst. Reaction condition: $10 \mathrm{mg}$ catalyst, $50 \mathrm{mg}$ benzyl alcohol, $1 \mathrm{~atm} \mathrm{O}_{2}, 100^{\circ} \mathrm{C}$. The used catalyst is recycled twice (at reaction conditions specified in Figure 2 caption) before the time conversion measurement.

\section{Top View}

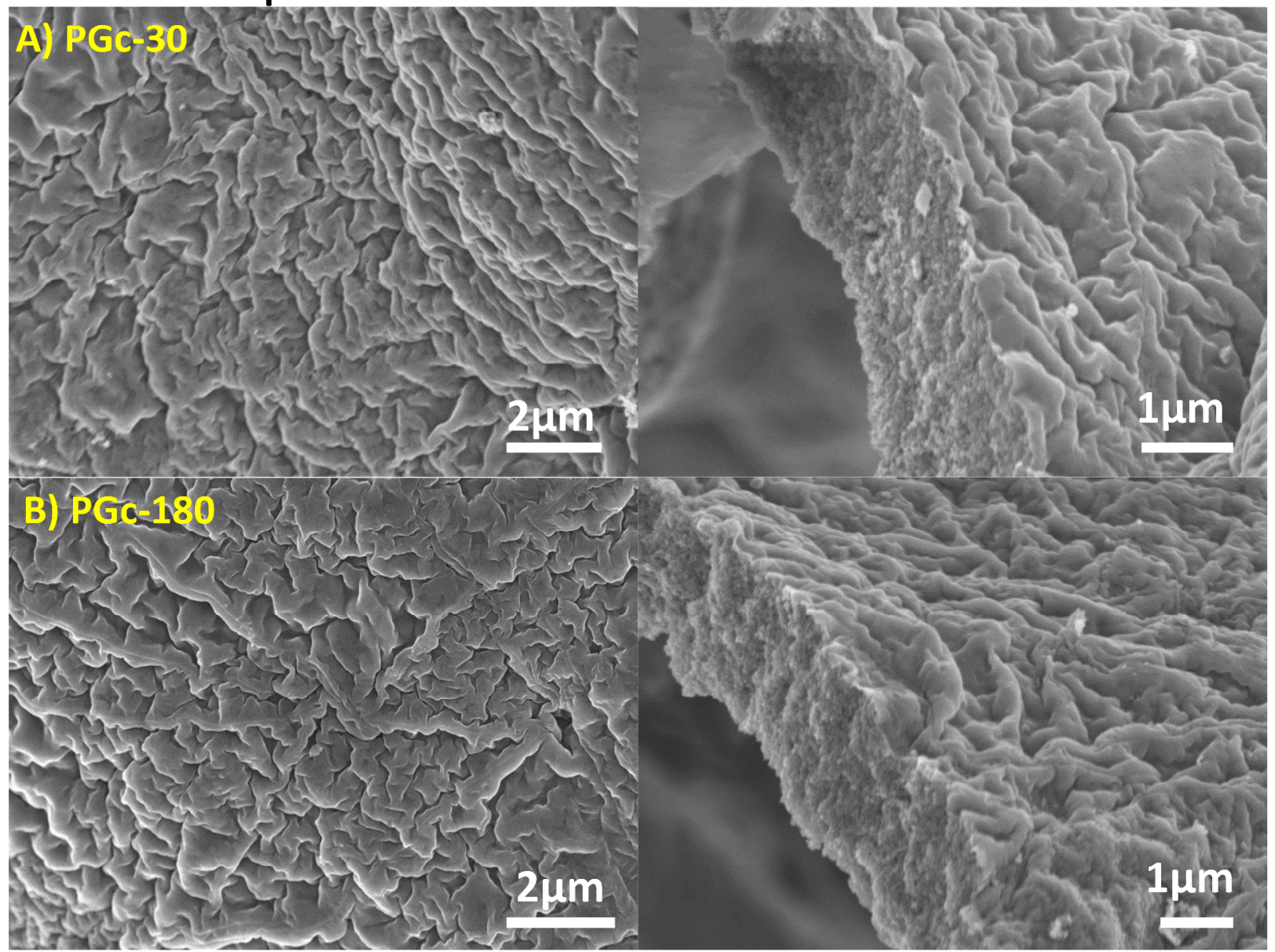

Figure S9. Scanning electron microscope image of the fabricated PGc-30 and PGc-180 catalysts. 


\section{PF-KPFM measurement of PGc catalysts:}

To understand the mechanism of the alcohol oxidation reaction catalyzed by PGc materials, we have conducted a PeakForce Kelvin probe force microscopy $\left(\mathrm{PF}_{\mathrm{K}} \mathrm{KPFM}^{\mathrm{TM}}\right)$. PF-KPFM $^{\mathrm{TM}}$, the combination of PeakForce Tapping mode and frequency modulated KPFM (FM-KPFM), integrates the benefits and capabilities of PeakForce Tapping and the superior spatial resolution and accuracy of FM-KPFM. PF-KPFM has the best performance of KPFM working in a dual-pass fashion. ${ }^{3}$ By using KPFM, one can measure the local surface potential of nanoscale materials, concurrently imaging their topography. Since KPFM measures the voltage required to nullify the work function $(\varphi)$ difference between the conductive tip and the sample $\left(\varphi_{\text {tip }}-\varphi_{\text {sample }}\right)$ or vice versa, depending on whether the potential was applied to the sample or the probe) the contrast in the contact potential difference (CPD) is equivalent to the local work function variation of the sample on a supporting substrate. So the local surface potential can be used to calculate the work function of the materials, if the work function of the tip is known. KPFM has been widely used to investigate the influence of dopants or atomic scale defects on the variation of work function. It has also been used to study the work function of graphene as a function of number of layers and heteroatomic doping. ${ }^{4-6}$

PF-KPFM measurements on the PGc materials were conducted with a Dimension ICON AFM setup inside an Argon-filled glove box where both $\mathrm{H}_{2} \mathrm{O}$ and $\mathrm{O}_{2}$ levels were below $0.1 \mathrm{ppm}$. The probes used were PFQNE-AL (Bruker AFM Probes), composed of a silicon nitride cantilever with a sharp silicon tip. The inert environment helped us to obtain more accurate measurements, since the dipole moment of any absorbed species can directly induce a difference in contact potential and, subsequently, a phase shift of our samples. ${ }^{7}$ To ascertain the accuracy of our surface potential measurements, the CPD measurement was first conducted for a piece of freshly cleaved highly ordered pyrolytic graphite (HOPG) in the glove box. The average CPD value of $0.60 \mathrm{~V}$ (with the standard deviation of $0.02 \mathrm{~V}$ ) was obtained from the measurements at four different spots on the same HOPG. Knowing the work function of the probe tip from previous experiments $(4.08 \mathrm{eV})$, the average work function $\varphi$ of HOPG was calculated to be $4.68 \mathrm{eV}$. This value is in agreement with the literatures. ${ }^{8}$ To measure the work function of PGc and PGc-180 with PF-KPFM, we first break the monolith of PGc and PGc-180 to small particles and disperse them into water or ethanol using bath sonication for 3 minutes. Then the samples for CPD measurements were prepared by drop casting the PGc and PGc-180 particles onto a doped silicon ( $\mathrm{Si}$ ) substrate with $50 \mathrm{~nm} \mathrm{SiO}$ layer. Because the PGc or PGc180particles only partially covers the $\mathrm{Si}$ substrate, the measured $\mathrm{CPD}$ value of $\mathrm{Si}$ substrate can be used as the reference value to calculate the work functions of PGc or PGc-180.

Figure S2 shows the topographical and CPD images simultaneously taken on the PGc and PGc-180, respectively. From the AFM images, we found that upon sonication treatment, the unique sandwich structure of PGc and PGc-180 was separated to graphene sheet like structures and irregular particles, which possibly from the porous monolith 
sandwiched between the sheets. The CPD for the sheets of PGc and PGc-180 is -287.71 $\mathrm{mV}$ and $-173.03 \mathrm{mV}$, respectively, from which we calculate the work function of PGc is $4.87 \mathrm{eV}$, which is $120 \mathrm{meV}$ higher than that of PGc-180(4.75 eV). Noted that the dark contrast observed in the CPD image indicates their work function is higher than the $\mathrm{Si}$ substrate used in this work. We also calculated the work function of the Si substrate is $4.58 \mathrm{eV}$, which is consistent with the values $(4.60-4.85 \mathrm{eV})$ reported in literatures, further demonstrating the accuracy of the measurements. By conducting PF-KPFM with several samples, which have particles of different sizes we found that the work function of PGc particles barely changes with their height. The average work function is $4.78 \mathrm{eV}$, slightly lower than that of the corresponding sheets. However, for the PGc-180, the work function dramatically changes with the height of the particles. The higher ones have lower work functions. The lowest work function measured is $3.3 \mathrm{eV}$ with an average particle height of $400 \mathrm{~nm}$, which is dramatically lower than that of PGc. Even though we still have difficulty to explain these results, they unambiguously demonstrated that the PGc-180 has much lower work functions.
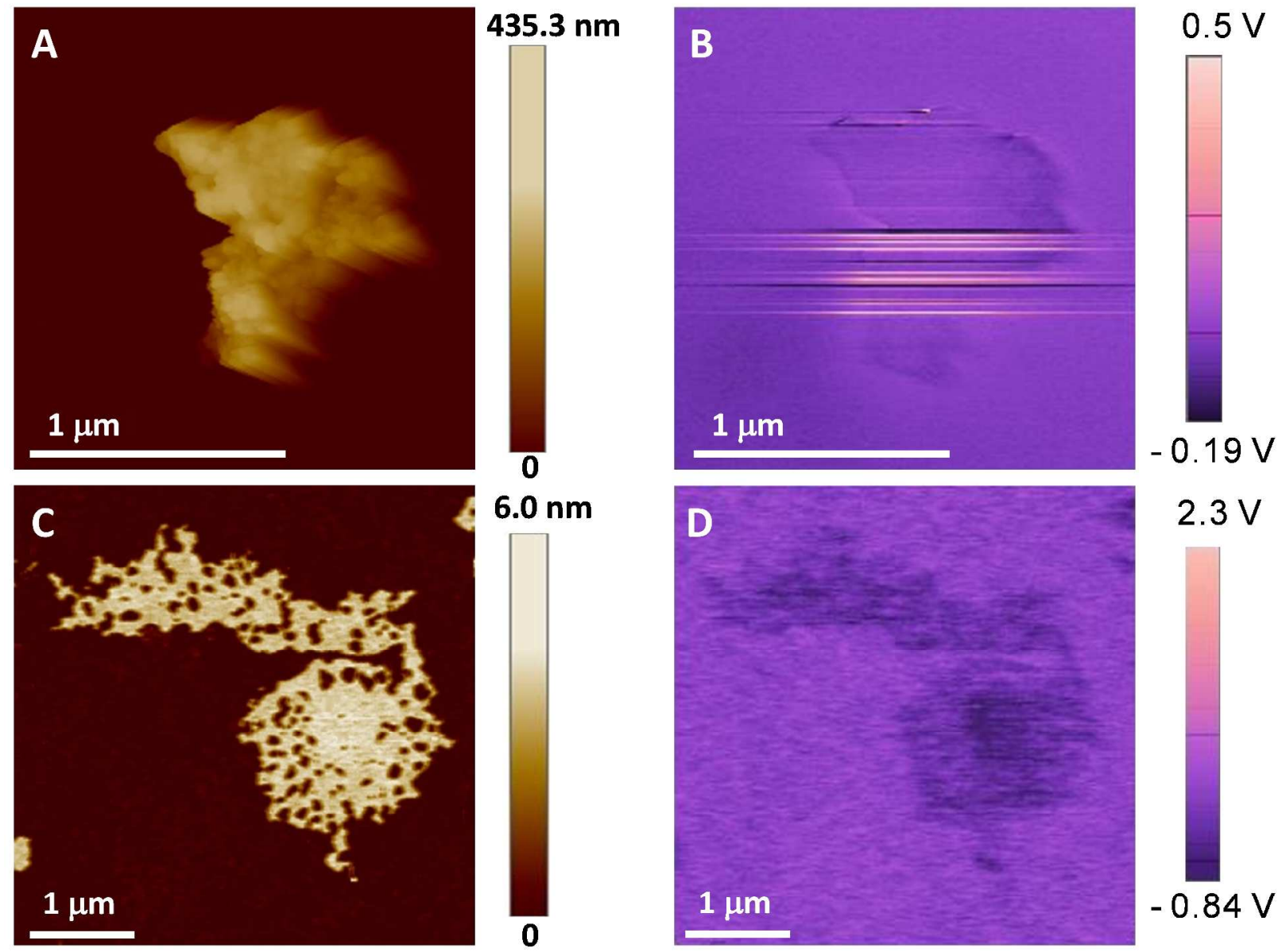

$6.0 \mathrm{~nm}$
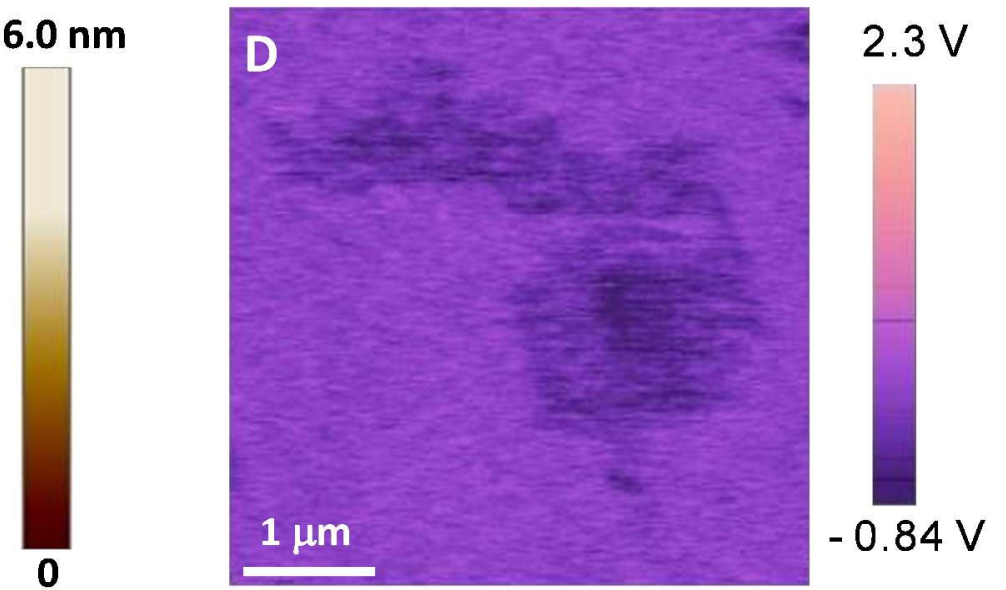

Figure S10. (A, C) AFM Topography and (B, D) PF-KPFM images for the PGc and the PGc180 catalysts. 


\section{X-ray fluorescence spectroscopic (XRF) measurement of PGc (fresh and used):}

To calculate \% P in PGc, XRF measurement was carried out using Horiba XGT-1000WR instrument with a high purity Si detector. The X-ray tube and current parameters were set to $50 \mathrm{kV}$ and $1 \mathrm{~mA}$. The standard samples were prepared by mixing known weight of ammonium phosphate with rGO and analyzed using XRF. The intensity versus \% $\mathrm{P}$ plotted to get linear calibration curve (Figure S11). After that, using the slope and intercept, we can calculate \% P presence in unknown samples (PGc fresh and used). The calculated \% P in fresh PGc and used PGc catalyst is to be 2.3 atomic \% (or 7.9 wt \%) and 2.6 atomic \% (or $9.5 \mathrm{wt} \%$ ).

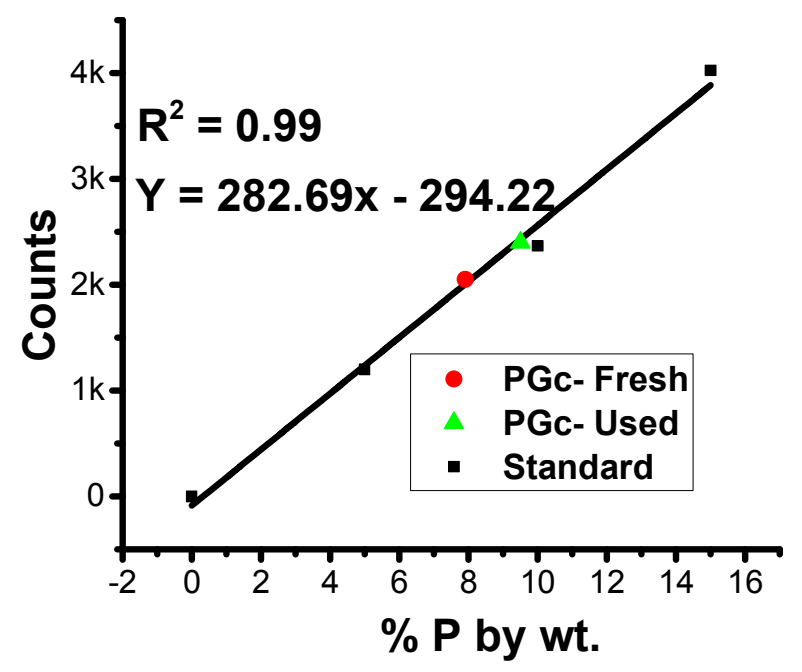

Figure S11. X-ray fluorescence spectroscopic (XRF) analysis of standard mixture (rGO with different $\% \mathrm{P}$ ). The used catalyst is recycled twice (at reaction conditions specified in the caption of Figure 2) before XRF measurement. 


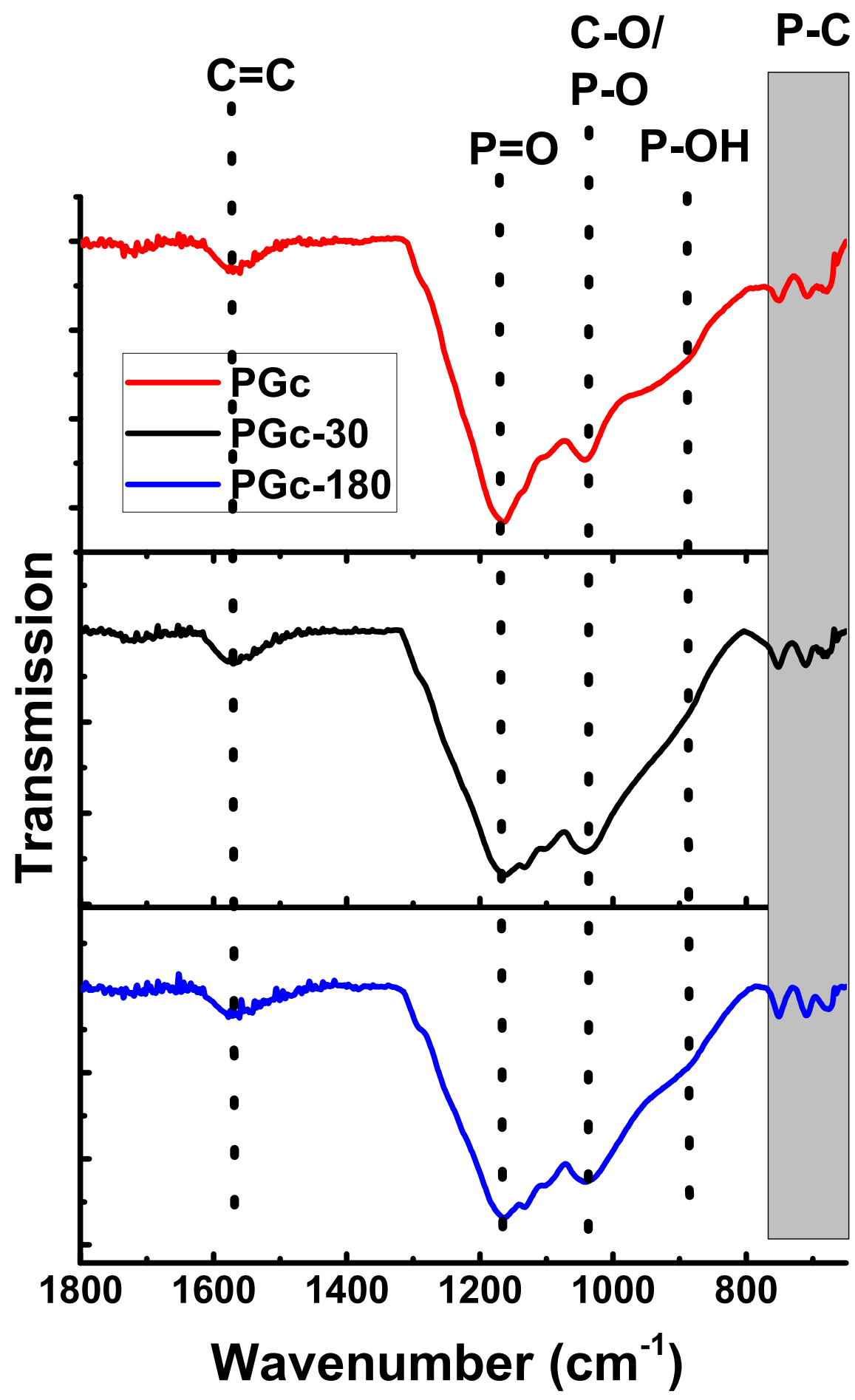

Figure S12. The FT-IR spectrum of PGc, PGc-30 and PGc-180 catalysts. 


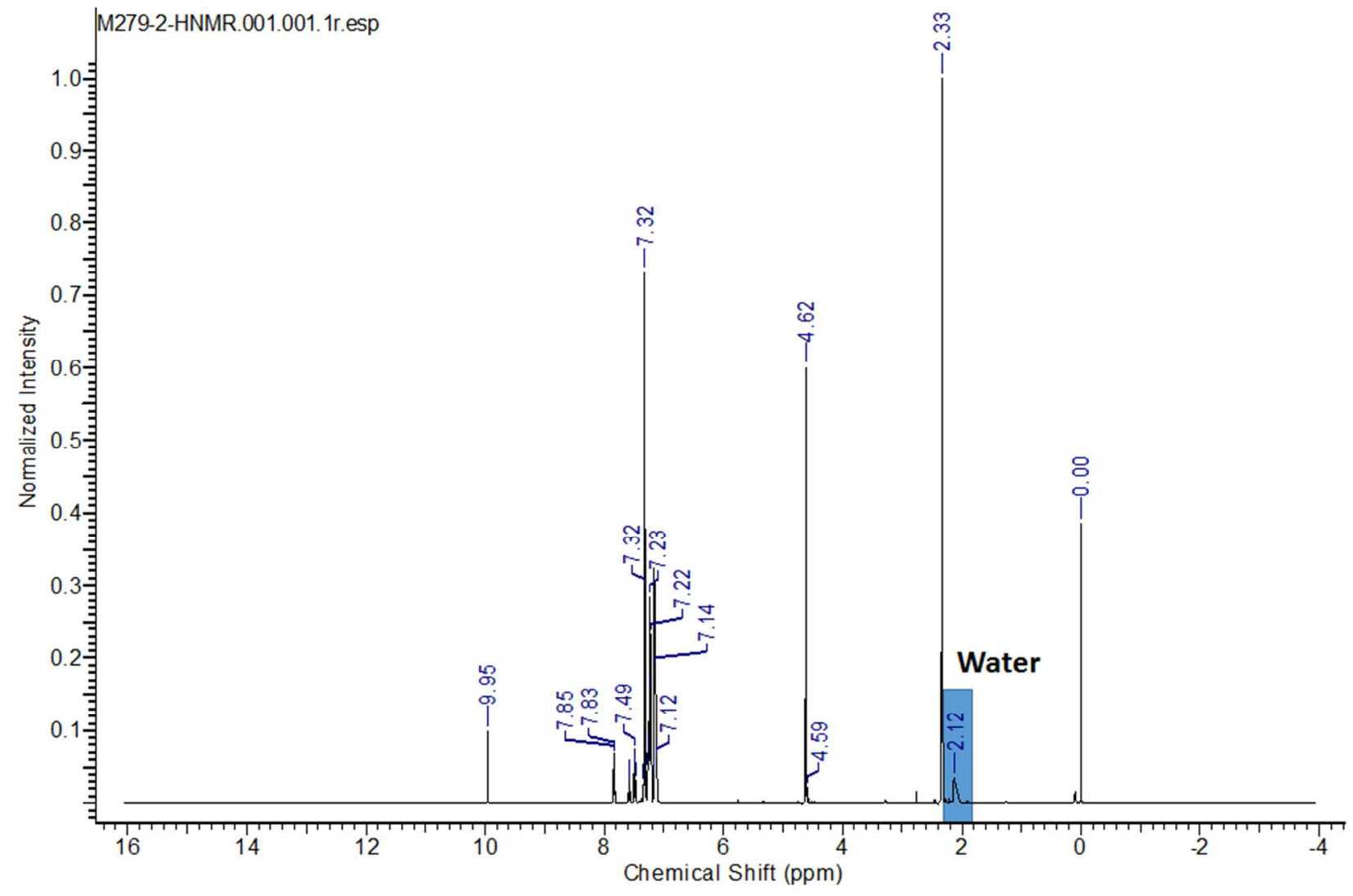

Figure S13. H-NMR spectrum of reaction mixture (Table 2- entry no. 4) containing benzyl alcohol (2H, $4.62 \mathrm{ppm})$, Benzaldehyde (1H, $9.95 \mathrm{ppm})$ and trace amount of water (2.12 ppm). 


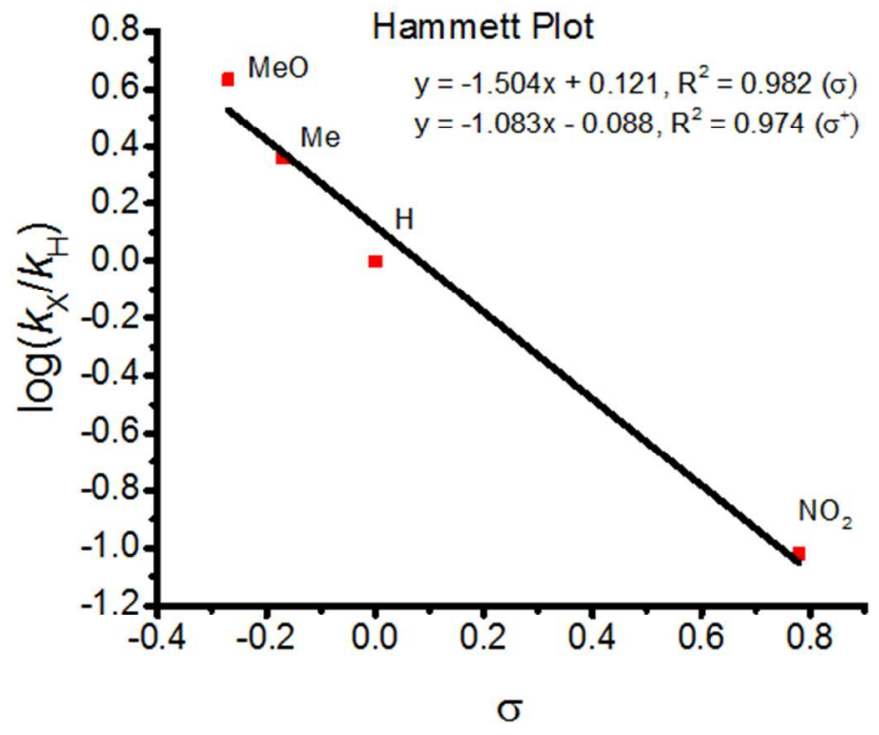

Figure S14. Hammett plot of Plot of log k vs. $\sigma$ for the oxidation of 4-substituted benzyl alcohols with PGc catalyst. 


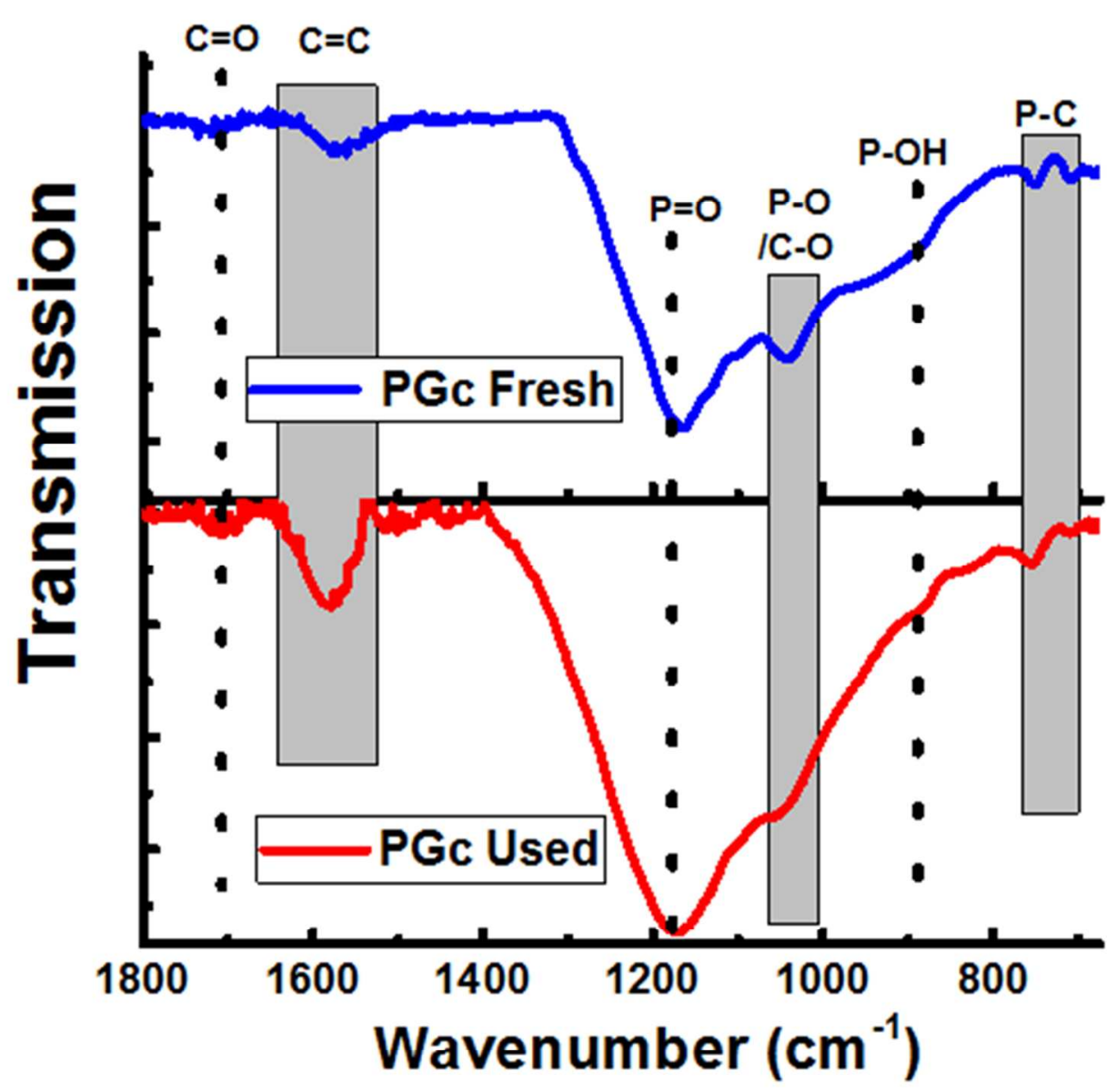

Figure S15. The FT-IR spectrum of the fresh and used PGc catalyst. The used catalyst was recycled twice (the reaction conditions were specified in Figure 2 caption) before this FT-IR measurement.

\section{References:}

1. Hu, F.; Patel, M. A.; Luo, F.; Flach, C. R.; Mendelsohn, R.; Garfunkel, E.; He, H.; Szostak, M. Graphene-Catalyzed Direct Friedel-Crafts Alkylation Reactions: Mechanism, Selectivity and Synthetic Utility. J. Am. Chem. Soc. 2015, 137, 14473-14480.

2. Brunauer, S.; Emmett, P. H.; Teller, E. Adsorption of Gases in Multimolecular Layers. $J$. Am. Chem. Soc. 1938, 60, 309-319.

3. Li, C; Minne, S.; Hu, Y.; Ma, J.; He, J.; Mittel, H.; Kelly, V.; Erina, N.; Guo, S.; Mueller, T. PeakForce Kelvin Probe Force Microscopy, Application Note \#140; Bruker Corporation: 2013. 
4. Baumgart, C.; Helm, M.; Schmidt, H. Quantitative Dopant Profiling in Semiconductors: A Kelvin Probe Force Microscopy Model. Phys. Rev. B: Condens. Matter Mater. Phys. 2009, 80, 085305 .

5. Koren, E.; Berkovitch, N.; Rosenwaks, Y. Measurement of Active Dopant Distribution and Diffusion in Individual Silicon Nanowires. Nano Lett. 2010, 10, 1163-1167.

6. $\quad$ Ziegler, D.; Gava, P.; Güttinger, J.; Molitor, F.; Wirtz, L.; Lazzeri, M.; Saitta, A.; Stemmer, A.; Mauri, F.; Stampfer, C. Variations in the Work Function of Doped Single-And Few-Layer Graphene Assessed by Kelvin Probe Force Microscopy And Density Functional Theory. Phys. Rev. B: Condens. Matter Mater. Phys. 2011, 83, 235434.

7. Lägel, B.; Baikie, I.; Petermann, U. A Novel Detection System for Defects and Chemical Contamination in Semiconductors Based upon the Scanning Kelvin Probe. Surf. Sci. 1999, 433, 622-626.

8. Beerbom, M.; Lägel, B.; Cascio, A.; Doran, B.; Schlaf, R. Direct Comparison of Photoemission Spectroscopy and in Situ Kelvin Probe Work Function Measurements on Indium Tin Oxide Films. J. Electron. Spectrosc. Relat. Phenom. 2006, 152, 12-17. 